\title{
Photonic-Chip-Enabled 25 Tb/s Optical Superchannel using Cyclic Spectra
}

Corcoran, Bill; Geng, Zihan; Rozental, Valery; Zhuang, Leimeng; Lillieholm, Mads; Lowery, Arthur J.

Published in:

Proceedings of 2017 European Conference on Optical Communication (ECOC)

Link to article, DOI:

10.1109/ECOC.2017.8345852

Publication date:

2017

Document Version

Peer reviewed version

Link back to DTU Orbit

Citation (APA):

Corcoran, B., Geng, Z., Rozental, V., Zhuang, L., Lillieholm, M., \& Lowery, A. J. (2017). Photonic-Chip-Enabled $25 \mathrm{~Tb} / \mathrm{s}$ Optical Superchannel using Cyclic Spectra. In Proceedings of 2017 European Conference on Optical Communication (ECOC) (pp. 3 pp.). IEEE. https://doi.org/10.1109/ECOC.2017.8345852

\section{General rights}

Copyright and moral rights for the publications made accessible in the public portal are retained by the authors and/or other copyright owners and it is a condition of accessing publications that users recognise and abide by the legal requirements associated with these rights.

- Users may download and print one copy of any publication from the public portal for the purpose of private study or research.

- You may not further distribute the material or use it for any profit-making activity or commercial gain

- You may freely distribute the URL identifying the publication in the public portal 


\title{
Photonic-Chip-Enabled $25 \mathrm{~Tb} / \mathrm{s}$ Optical Superchannel using Cyclic Spectra
}

\author{
Bill Corcoran ${ }^{(1,2)}$, Zihan Geng ${ }^{(1,2)}$,Valery Rozental(1), Leimeng Zhuang ${ }^{(1)}$, Mads Lillieholm ${ }^{(3)}$, \\ Arthur J. Lowery ${ }^{(1,2)}$
}

(1) Monash Electro-Photonics Laboratory, Department of Electrical and Computer Systems Engineering, Monash University, Australia bill.corcoran@monash.edu

(2) Centre for Ultrahigh-bandwidth Devices for Optical Systems (CUDOS), Australia

(3) DTU Fotonik, Denmark

Abstract We demonstrate the all-optical generation of a 3.8-THz wide superchannel, using a photonicchip-based filter for sub-channel definition. The photonic chip is able to shape and aggregate $304 \times N R Z$ 32-QAM sub-channels, carrying 10-Gbd data, with an effective data-rate of $24.79 \mathrm{~Tb} / \mathrm{s}$.

Optical superchannels enable the full use of the lightwave communications spectrum, potentially enabling enhancement of spectral utilization beyond the $64 \%$ currently used for $100 \mathrm{~Gb} / \mathrm{s}$ PM-QPSK channels, or proposed for future 400G PM-16QAM channels. The generation of superchannels can be achieved through signal shaping in either the digital domain through the use of high-precision digital-to-analogue converters ${ }^{12}$, or in optical domain by filtering a modulated optical wave ${ }^{345}$.

Generating superchannels through optical filtering may be an attractive alternative to digital pulse shaping, as the drive signals imprinting data onto an optical carrier need only a few amplitude levels, as opposed to digitally shaped signals that require many bits of precision. However, the optical filters required for superchannel generation can be difficult to realize, and at the receiver side prior demonstrations of OFDM have either required a large oversampling ratio in digital processing ${ }^{4}$ or a sophisticated optical front end in a fully dispersion compensated link ${ }^{3}$.

We have previously demonstrated that a photonic chip implementation of a ring-assisted MachZehnder interferometer (RAMZI) can provide filter shapes similar to a digital $8 \%$ root-raised-cosine (RRC) filter, to produce Nyquist wavelength division multiplexing (NWDM) like signals ${ }^{5}$. This has recently been shown to operate over the full C-band ${ }^{5}$, which supports highly parallel processing, simultaneously providing signal shaping to many wavelength channels.

'Cyclic spectra' signals provide a new candidate for optical filter based superchannels ${ }^{6}$. We recently proposed and demonstrated this format, where the signal band includes some redun- dant, repeated spectral information, to provide resilience against impairments arising from wavelength routing in optically switched networks ${ }^{6}$. This new format requires similar pulse shaping to NWDM, and so is an obvious candidate for an optical filter based implementation using a photonic-chip-based RAMZI ${ }^{5}$.

In this paper, we show that 10-Gbd cyclic spectra sub-channels with $20 \%$ spectral redundancy (12.5-GHz wide) can be carved from a single 32-QAM modulated, 3.8-THz wide optical comb source, overall providing a $24.8 \mathrm{~Tb} / \mathrm{s}$ optical super-channel. We test each sub-band, and show error-free reception of 297 separate sub bands. This shows, for the first time, the ability to all-optically shape and multiplex high-order QAM signals into a single multi-terabit superchannel, while allowing sub-band reception with a standard coherent receiver processing at 2 samples/symbol. We further test a subset of sub-bands after transmission in 20-km long metropolitan-area field-trial link, and again show error-free performance. This demonstration shows the potential for optical filter based superchannels to provide massive parallel processing to enable ultra-high data rates in metro-area optical networks.

The concept of generating cyclic-spectra signals through optical filtering is shown in Fig. 1. Here, we use an optical comb to provide a bank of optical carriers, separated by the repetition rate of a mode-locked laser (MLL). By modulating this comb with data with a symbol rate $\left(R_{S}\right)$ matched to the MLL repetition rate, the comb lines are broadened resulting in a 'white' optical spectrum (Fig. 1b) ${ }^{4}$

This white spectrum can be filtered anywhere 

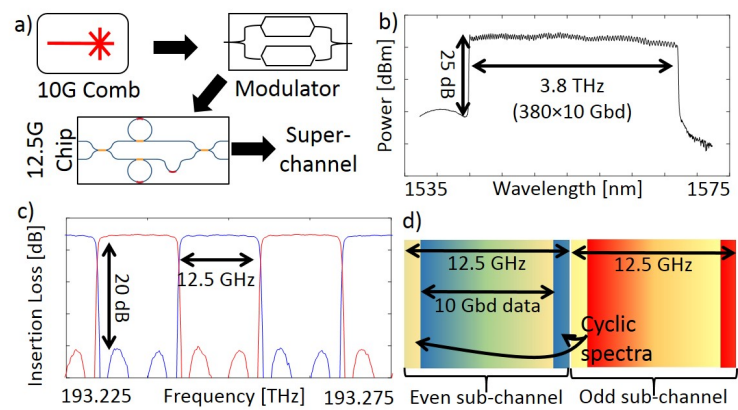

Fig. 1: a) Simplified superchannel generation schematic, b) Spectrum of $10 \mathrm{Gbd}$ modulated optical frequency comb, c) Photonic chip filter response, d) Illustration of resulting cyclic spectra superchannel structure

along its extent to provide a channel carrying the modulated data. To generate a cyclic-spectrum signal from the modulated comb, we pass the signal through a RAMZI with a pass-band $(\Delta f)$ in excess of the signal symbol rate (Fig. 1c). This will then produce a cyclic-spectrum sub-band with a spectral redundancy of $1-R_{S} / \Delta f$ (Fig. $1 d)$. Note that, since the pass-band and symbol rate are mis-matched, the optical comb lines will not necessarily line up with the center of the RAMZI-defined optical filters. As such, the central frequencies of the sub-bands are defined by these filters, rather than the MLL comb lines.

The experimental set-up we use to generate the cyclic-spectra super-channel is shown in Fig. 2. Pulses from a MLL (c.a. 2 ps duration) with a repetition rate of $10.015 \mathrm{GHz}$ are amplified and fed into a normally dispersive highly nonlinear fiber (HNLF, D $=-0.5 \mathrm{ps} /(\mathrm{nm} . \mathrm{km}), \gamma=11 \quad(\mathrm{~W} . \mathrm{km})^{-1}$ at $1550 \mathrm{~nm}$ ). The resulting comb is then processed line-by-line with a wavelength selective switch (WSS), to equalize tone powers and truncate the spectrum to $3.8 \mathrm{THz}$, before modulation. The $20-\mathrm{GHz}$ bandwidth $\mathrm{LiNbO}_{3}$ modulator (CMZM) is driven by an arbitrary waveform generator (AWG) running at $90.135 \mathrm{GSa} / \mathrm{s}$, programmed to generate NRZ-32-QAM at $10.015 \mathrm{Gbd}$ (i.e. matched to the repetition rate of the MLL). The resulting modulated comb is then fed to an input port of the ring-assisted Mach-Zehnder interferometer (RAMZI) chip ${ }^{5}$, which shapes the spectrum into odd-and-even sub-channels spaced by 12.5 $\mathrm{GHz}$. This shaping provides a $20 \%$ redundancy cyclic spectrum signal in each sub-band. The odd-and-even bands are delay decorrelated, and combined to form a 3.8-THz wide optical superchannel. Polarization multiplexing is then emulated by delay decorrelation using a 3-dB coupler and polarization beam splitter. The signal is then either passed directly to the receiver for

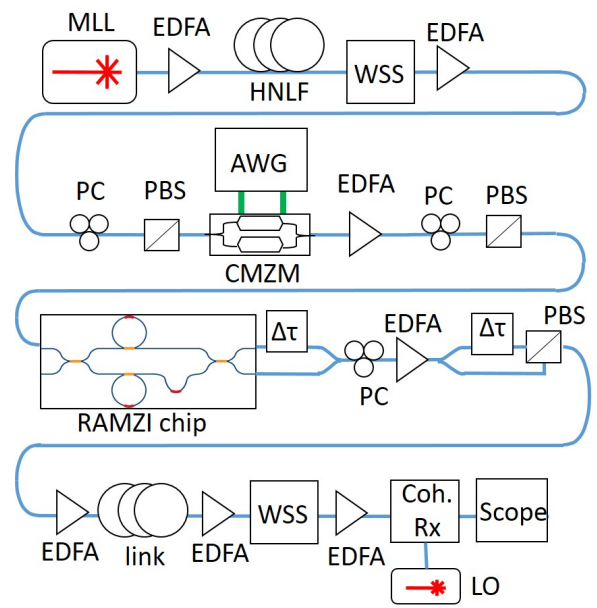

Fig. 2: Experimental set-up. PC: Polarization controller, PBS: Polarization beam splitter, $\Delta \tau$ : optical delay line.

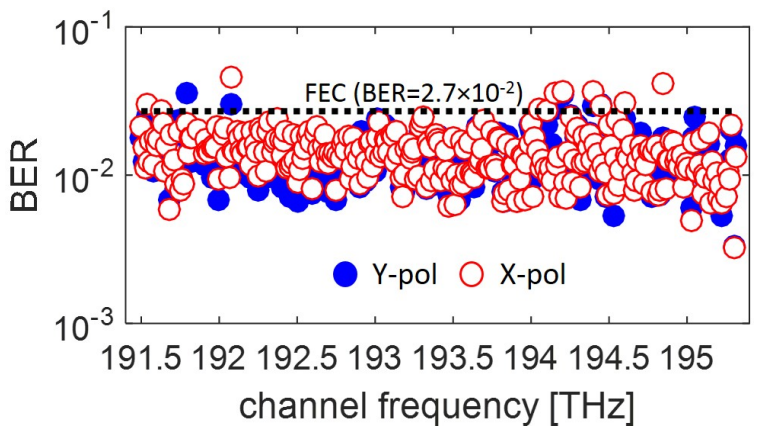

Fig. 3: BER for all 608 sub-channels (304 frequencies, 2 polarizations) in back-to-back configuration.

testing, or through a 20-km loop-back of installed fiber between Monash University's Caulfield and Clayton campuses.

The receiver uses a $20-\mathrm{GHz}$ bandwidth drop filter to isolate sub-bands before coherent detection with a 25-GHz bandwidth integrated receiver (Coh. Rx), sampled with an 80-GSa/s real-time oscilloscope. The signal is processed offline at 2 $\mathrm{Sa} /$ symbol (20.03 GSa/s), using a spectral peak search frequency offset algorithm, followed by frame synchronization, two step equalization with a standard constant modulus algorithm equalizer initializing a radial decision directed equalizer, maximum likelihood phase estimation then biterror counting. For bit-error rate measurement, we assume the use of a $20 \%$ overhead LDPC-CC forward-error correction (FEC) $\mathrm{code}^{7}$, defining an error free threshold at $\mathrm{BER}=2.7 \times 10^{-2}$. Fig. 3 shows the measured BER for all 608 (304 frequencies, 2 polarizations) sub-channels in back-to-back configuration. 594 channels clear the FEC threshold, providing a net data rate of $24.78 \mathrm{~Tb} / \mathrm{s}(10.015 \times 5 \times 608 / 1.2 \mathrm{~Gb} / \mathrm{s})$. As observed in Fig. 3, there is no clustering of failed channels, indicating that the performance of channel across the C-band is relatively even. 


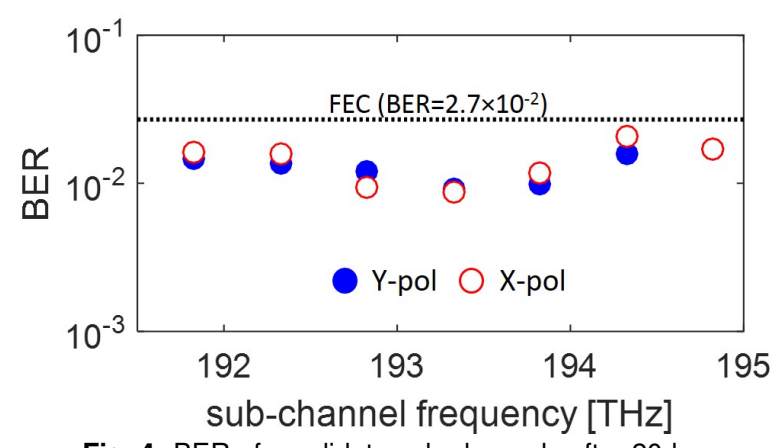

Fig. 4: BER of candidate sub-channels after 20-km transmission through field installed fibers

To show that the generated superchannel can be transmitted over useful distances, we launch into a 20-km loop-back of field installed standard single mode fiber, with a round-trip loss of 5.8-dB. We analyze a representative sub-set of subchannels at 7 different frequencies, spaced by $500 \mathrm{GHz}$, and show negligible penalty compared to back-to-back (Fig. 4). This indicates that the generated all-optical superchannel is compatible with transmission through installed metro-area fibers.

We next analyze the limitations to performance of the all-optical superchannel generation system. By measuring the performance of a sub-band centered at $193.25 \mathrm{THz}$, with and without the chip in place for channel shaping, and with and without the $20-\mathrm{km}$ link. Fig. 5 shows constellation diagrams and associate $\mathrm{Q}$-factors, with $\mathrm{Q}$ extracted from error vector magnitude (EVM) as $Q=20 \log _{10}\left(1 / E V M^{2}\right)$. Fig. 5a shows that the modulated comb is limited to 18.3-dB Q, which we attribute to a low per-tone optical power, which reduces the signal-to-noise directly out of the modulator, as well as some modulator nonlinearity. Fig. $5 \mathrm{~b}$ includes a digitally implemented $12.5 \mathrm{GHz}$ wide, $8 \% \mathrm{RRC}$ filter at the receiver, to emulate the best-case filtering performance for the RAMZI. This shaping drops the performance by about $1 \mathrm{~dB}$ to $\mathrm{Q}=17.4 \mathrm{~dB}$. When the chip is added back into the system, we measure a sub-band performance of $\mathrm{Q}=16.5 \mathrm{~dB}$ (Fig. 5c), which we attribute to the relatively high loss of the chip (c.a. $12 \mathrm{~dB}$ fiber-to-fiber) and passive cross-talk between channels. This indicates that the chip itself provides $<2 \mathrm{~dB}$ penalty when providing simultaneous sub-channel shaping and multiplexing. Finally, Fig. 5d shows the constellation after $20-\mathrm{km}$ installed fiber transmission, essentially showing no further penalty above back-to-back transmission.

In conclusion, we demonstrate the ability to use a photonic chip based interleaver to simultane-

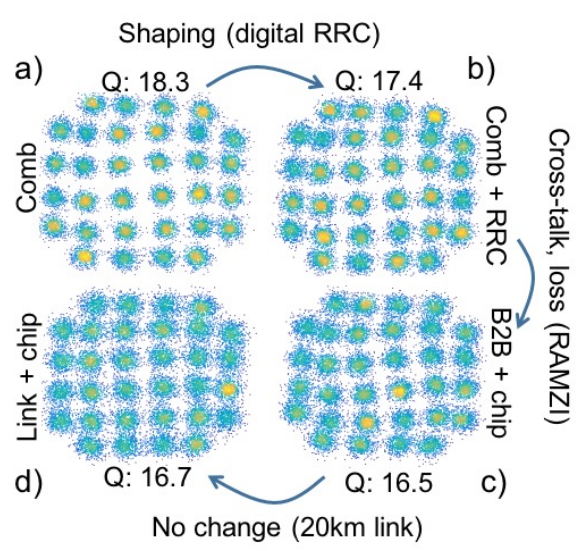

Fig. 5: Evolution of performance penalties in the superchannel system.

ously provide channel shaping and multiplexing, to produce a superchannel with an aggregate data rate of $24.79 \mathrm{~Tb} / \mathrm{s}$. Analyzing the penalties associated with generation, we find that the chip induces $<2 \mathrm{~dB}$ penalty in back-to-back, and we show negligible penalty after transmission over installed metropolitan area fiber. This demonstration shows the potential for such photonic chips to provide for passive aggregation of multiple independent channels into a single optical superchannel for future ultra-high density WDM systems, toward fully utilizing the available optical communications spectrum in installed fiber links.

We thank Lars Grüner-Nielsen of OFS (now with Danish Optical Fiber Innovation) for the comb-broadening HNLF. Funding: Australian Research Council (ARC) (CE110001018, FL130100041)

\section{References}

[1] W. Sheih et al., "Coherent optical orthogonal frequency division multiplexing," Electron. Lett., Vol. 42, no. 10 (2006).

[2] G. Bosco et al., "Performance Limits of Nyquist-WDM and CO-OFDM in High-Speed PM-QPSK Systems," J. Lightwave Tecnol., Vol. 22, p. 1129 (2010).

[3] D. Hillerkuss et al., "26 Tbps line-rate super-channel transmission utilizing all-optical fast Fourier transform processing," Nat. Photon., Vol. 5, p. 364 (2011).

[4] J. Schroeder et al., "All-optical OFDM with cyclic prefix insertion using flexible wavelength selective switch optical processing,' 'J. Lightwave Technol., Vol. 32, p. 752 (2014).

[5] Z. Geng et al., "Full C-band Nyquist-WDM Interleaver Chip," Proc. OFC, Tu2l.4, Los Angeles (2017).

[6] B. Corcoran et al., "Cyclic spectra for wavelength-routed opticalnetworks," Opt. Lett., Vol. 42, p. 1101 (2017).

[7] D. Chang et al., "LDPC convolutional codes using layered decoding algorithm for high speed coherent optical transmission,", Proc. OFC, OW1H.4, Los Angeles (2012). 